\title{
QuickSun 830A module solar simulator. Study of mini PV modules
}

\author{
Malgorzata Pociask-Bialy ${ }^{1,}$, Radoslaw Maciejko ${ }^{1}$ \\ ${ }^{1}$ Faculty of Mathematics and Natural Sciences, University of Rzeszow, 35-959 Rzeszow, Poland
}

\begin{abstract}
QS830A module solar simulator is applied to measure electrical parameters of standard PV modules based on mono/polycrystalline silicon solar cells, large format photovoltaic (PV) modules $150 \mathrm{~cm} \times 220 \mathrm{~cm}$, with effective measurements time of $2 \mathrm{~ms} / 4 \mathrm{~ms}$, flush pulse duration $3 \mathrm{~ms} / 10 \mathrm{~ms}$ (one flash tube / two flash tubes), and non-uniformity less than $2 \%$. In order to comply with the Class AAA tolerances of the standard IEC 60904-9 Edition 2.0 of QuickSun 830A simulators (Endeas Oy, Finland), proprietary optical system behind the light source filament has been developed for filtering spectrum and improving irradiance nonuniformity simultaneously. Non-uniformity parameter for $14 \%$ of total modules testing area, i.e. $0.65 \mathrm{~m}^{2}$, will be appointed in this work.
\end{abstract}

\section{Introduction}

Quick Sun 830A simulator, Endeas Oy, Finland, placed at University of Rzeszow was horizontally installed and instructions for constructing a corresponding flash tunnel were supplied with the simulators. A flash tunnel gives better access to the flash head e.g. for changing the flash tube. A test surface with fixed test sensor positions covering the nominal test area of each simulator is a ground and basic part in order to perform fluent and reproducible irradiance non-uniformity measurements. After changing the xenon flash lamp (light source) such tests must be obligatorily performed. Non-uniformity measurements, normally, must be provided minimum once per half year with testing at our Renewable Energy Sources laboratory at the Center for Innovation and Transfer of Natural Science and Engineering Knowledge of the University of Rzeszow (we are testing more than 300 modules per month). At a factory conditions testing capacity for QS830A is 90 measurements per hour. All information about parameters and measurement accuracies are presented on the producer site [1,2]. Presented here are investigations that were done during non-uniformity tests of QS 830A. Uniformity tests were always provided after changing the flush tube.

The goal of that research was to show that even if the condition for uniformity QS830A class A is not satisfied for whole testing area, we can distinguish a smaller area that meets class A requirements for inhomogeneity of light intensity, i.e. $2.28 \mathrm{~m}^{2}, 2.20 \mathrm{~m}^{2}, 1.14 \mathrm{~m}^{2}$, $0.65 \mathrm{~m}^{2}$. The reduced area is perfectly suited for the certification tests of mini photovoltaic's modules. Measurements are made under standard test conditions (STC): $1000 \mathrm{~W} / \mathrm{m}^{2}, 25^{\circ} \mathrm{C}, \mathrm{AM} 1.5 \mathrm{G}$ presented in [3-6].

* Corresponding author: pociask@ur.edu.pl 


\section{Non-uniformity tests}

Spectrum of every simulator, of QS830A too, is recorded and compared to comply with the Class A tolerances as specified in IEC 60904-9 Edition 2.0 [2], irradiance and temperature correction procedures and coefficients are presented in IEC 891 Edition 2.0 [5]. Nonuniformity is measured by mapping the irradiance of the simulator beam over the test area, i.e. in case of QS830A by recording the short circuit current $\mathrm{I}_{\mathrm{SC}}$ distribution of a laminated c-Si cell type M2 No. NAPS 31292 (uniformity sensor, dimension $12.6 \mathrm{~cm} \times 12.4 \mathrm{~cm}$ ), over specified test area $[1,5]$. That method is used in all types of flush and pulsed simulators in which the source of light is xenon lamp. Same test at QS800 series simulators can be easily reproduced by using the test sensor and QuickSun software tools provided with the simulator.
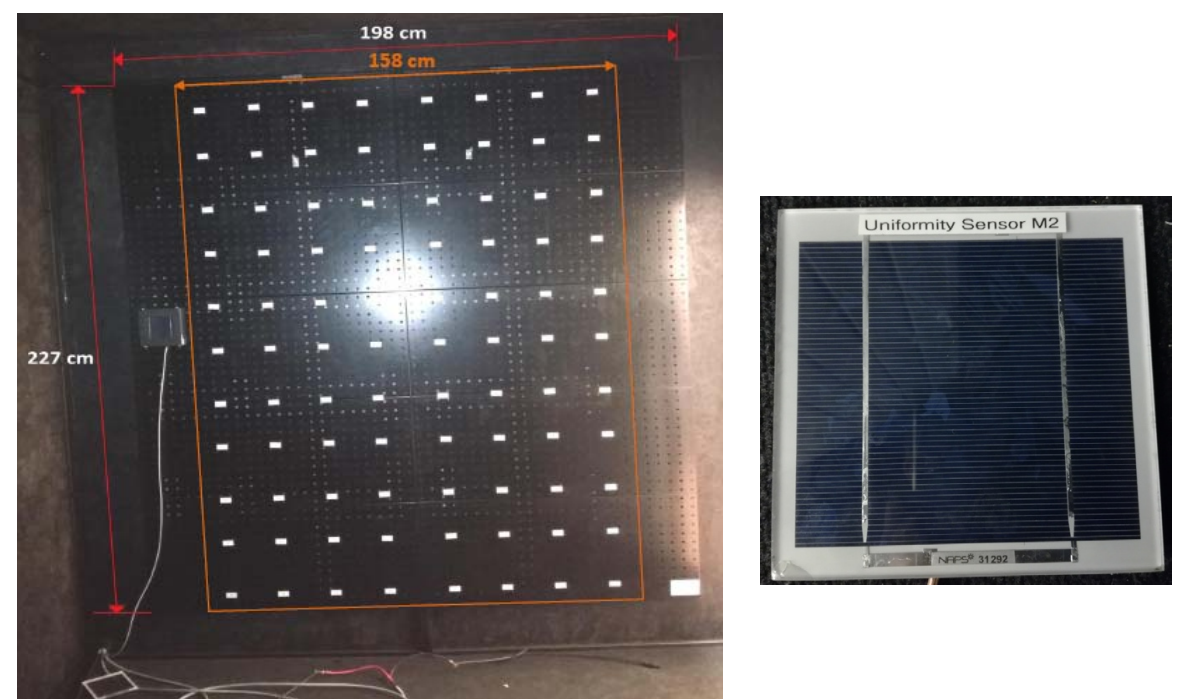

Fig. 1. Testing area $2.27 \mathrm{~m}$ x $1.58 \mathrm{~m}$ and uniformity sensor M2 No. NAPS 31292.

Table 1. Matrix of the simulator's maximum tested field, 8 columns x11 rows with segments numbering.

\begin{tabular}{|c|c|c|c|c|c|c|c|}
\hline 1.1 & 1.2 & 1.3 & 1.4 & 1.5 & 1.6 & 1.7 & 1.8 \\
\hline 2.1 & 2.2 & 2.3 & 2.4 & 2.5 & 2.6 & 2.7 & 2.8 \\
\hline 3.1 & 3.2 & 3.3 & 3.4 & 3.5 & 3.6 & 3.7 & 3.8 \\
\hline 4.1 & 4.2 & 4.3 & 4.4 & 4.5 & 4.6 & 4.7 & 4.8 \\
\hline 5.1 & 5.2 & 5.3 & 5.4 & 5.5 & 5.6 & 5.7 & 5.8 \\
\hline 6.1 & 6.2 & 6.3 & 6.4 & 6.5 & 6.6 & 6.7 & 6.8 \\
\hline 7.1 & 7.2 & 7.3 & 7.4 & 7.5 & 7.6 & 7.7 & 7.8 \\
\hline 8.1 & 8.2 & 8.3 & 8.4 & 8.5 & 8.6 & 8.7 & 8.8 \\
\hline 9.1 & 9.2 & 9.3 & 9.4 & 9.5 & 9.6 & 9.7 & 9.8 \\
\hline 10.1 & 10.2 & 10.3 & 10.4 & 10.5 & 10.6 & 10.7 & 10.8 \\
\hline 11.1 & 11.2 & 11.3 & 11.4 & 11.5 & 11.6 & 11.7 & 11.8 \\
\hline
\end{tabular}

Current, voltage and temperature measurement accuracies are calibrated and verified to comply with IEC 60904-1 Edition 2.0 specifications [3]. Irradiance measurement accuracy was factory calibrated but final calibration of QS830A was performed at Renewable Sources Laboratory by applying the two certified reference Si modules monocrystalline and 
polycrystalline. Test report power measurements of both PV modules were carried out at the Fraunhofer Institute for Solar Energy Systems ISE in Freiburg. Mono-Si type SFE.MF6-255, has a protocol No. NPC0002001NPC0613-V01, but poly-Si type SFE.PF-6-235 has a protocol No. NPC001001NPC0613-V01. Both protocols are performed on Pasan MFG 502 flash simulator in accordance to IEC60904-1 [3] and IEC60904-3 [4]. The area of $1.58 \mathrm{~m} \times 2.27 \mathrm{~m}$, seen on Fig. 1, on which the light intensity is determined, was particulary investigated and is divided into a grid of 88 measurement positions, i.e. 8 columns and 11 rows. The whole area is $3.59 \mathrm{~m}^{2}$. The experimental test screen on which the tested PV modules are mounted on special holders is slightly larger, as we can see on Fig. 1.

\subsection{Non-uniformity test. QS830A software}

Figure 2 shows matrix of non-uniformity measured with help of uniformity c-Si sensor M2 No. NPPS 31292 on the left, and a graphic distribution of illumination automatically generated by the QS830A software on the right. This distribution is obtained on the basis of the short-circuit current $\mathrm{I}_{\mathrm{SC}}[\mathrm{A}]$ distribution, as it was mentioned previously.

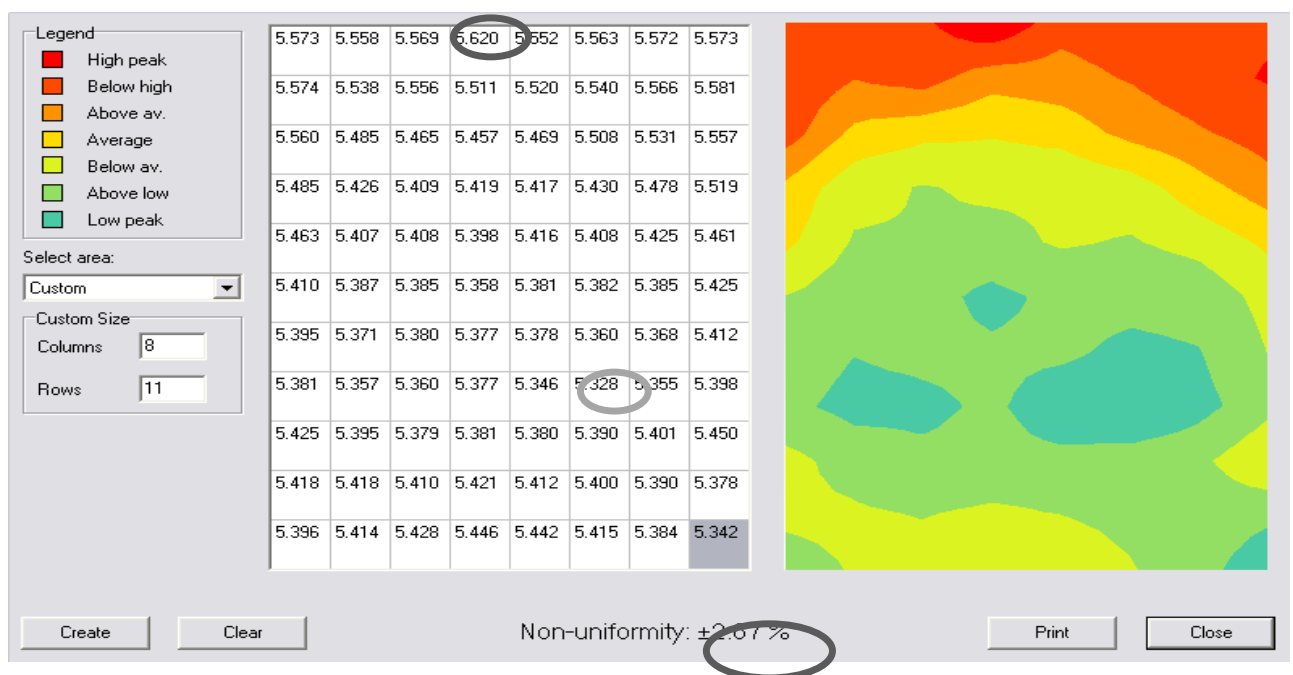

Fig. 2. Distribution of light intensity distribution resulting from simulator software obtained on the basis of the short-circuit current $\mathrm{I}_{\mathrm{SC}}[\mathrm{A}]$ distribution.

\section{Results and discussion}

\subsection{Non-uniformity test determining from I-V characteristics measured for uniformity c-Si cell}

Spatial distributions of short circuit for matrix 8 columns on 11 rows i.e. 88 tested points are show in Table 2.

The power output of the uniformity c-Si cell was measured using QS830A equipment. Following characteristics of the uniformity solar cell were evaluated: $\mathrm{I}_{\mathrm{SC}}$ - short circuit current $[\mathrm{A}], \mathrm{U}_{\mathrm{OC}}$ - open circuit voltage [A], MPP- maximum power point [W], FF - fill factor $[\%], \eta$ - energy conversion efficiency [\%]. Each current-voltage characteristics was repeated three times. 
Table 2. $\mathrm{I}_{\mathrm{SC}}[\mathrm{A}]$ average distribution of uniformity c-Si cell on whole tested screen with marked area of 8 columns $x 7$ rows.

\begin{tabular}{|l|l|l|l|l|l|l|l|}
\hline 5.573 & 5.560 & 5.570 & 5.620 & 5.554 & 5.564 & 5.572 & 5.574 \\
\hline 5.574 & 5.534 & 5.557 & 5.511 & 5.518 & 5.542 & 5.568 & 5.582 \\
\hline 5.556 & 5.483 & 5.465 & 5.456 & 5.469 & 5.506 & 5.533 & 5.560 \\
\hline 5.481 & 5.428 & 5.408 & 5.414 & 5.420 & 5.431 & 5.484 & 5.519 \\
\hline 5.458 & 5.408 & 5.410 & 5.400 & 5.413 & 5.410 & 5.422 & 5.465 \\
\hline 5.409 & 5.383 & 5.385 & 5.360 & 5.381 & 5.380 & 5.381 & 5.427 \\
\hline 5.369 & 5.369 & 5.380 & 5.375 & 5.377 & 5.358 & 5.373 & 5.412 \\
\hline 5.379 & 5.353 & 5.358 & 5.379 & 5.347 & & 5.356 & 5.400 \\
\hline 5.424 & 5.394 & 5.378 & 5.380 & 5.381 & 5.388 & 5.399 & 5.452 \\
\hline 5.418 & 5.415 & 5.411 & 5.423 & 5.411 & 5.399 & 5.387 & 5.381 \\
\hline 5.395 & 5.414 & 5.428 & 5.445 & 5.442 & 5.415 & 5.342 & 5.342 \\
\hline
\end{tabular}

In accordance with international standards for determining the parameter of the light intensity, in accordance with the generally accepted formula [5, 7-9] common for any type of module testers, we checked the correctness of calculations of the area we study:

$$
\Delta E_{Q S 830 A}=\left(E_{\max Q S}-E_{\min Q S}\right) \cdot 100 \% /\left(E_{\max Q S}+E_{\min Q S}\right)
$$

where $E_{\max Q S}$ is the maximum value of light intensity, $E_{\min Q S}$-the minimum value of light intensity. Results of non-uniformity performer for each of 88 average I-V characteristics is equal to $2.64 \%$, shown at row two of Table 3 . As we can see, the system software analyzed result is the same as presented in Figure 2, equal to 2.67\% (see: first row of Table 3).

Both results are in very good agreement, but the condition of non-uniformity for QS830A class AAA solar module tester is not met, because it must be less than $2 \%$.

Table 3. Results of calculation of non-uniformity parameter of QS830A performed on the basis of software intensity light distribution and distribution done on the basis of I-V characteristics for uniformity cell.

\begin{tabular}{|c|c|c|c|c|}
\hline & $\begin{array}{c}E_{\max Q s} \\
{[\mathrm{~A}]}\end{array}$ & $\boldsymbol{E}_{\min Q S}[\mathbf{A}]$ & $\begin{array}{c}\Delta E_{Q S 830 A} \\
{[1 / 1]} \\
\end{array}$ & $\begin{array}{c}\left(\Delta E_{\text {QS830A }} \pm\right. \\
\left.d\left(\Delta E_{\text {OS830A }}\right)\right)[\%]\end{array}$ \\
\hline $\begin{array}{l}\text { as a function of } \\
\text { QS830A software }\end{array}$ & 5.620 & 5.328 & 0.02667 & $2.670 \pm 0.003$ \\
\hline$\Delta E_{O S 830 A}, \mathbf{8}^{\mathbf{x}_{11}}$ & 5.620 & 5.331 & 0.02639 & $2.640 \pm 0.002$ \\
\hline
\end{tabular}

Results of $\mathrm{I}_{\mathrm{SC}}[\mathrm{A}]$ measurements with isotherms of $\mathrm{U}_{\mathrm{OC}}[\mathrm{V}], \mathrm{I}_{\mathrm{SC}}[\mathrm{A}], \mathrm{U}_{\mathrm{MP}}-$ voltage at maximum power point $[\mathrm{V}], \mathrm{I}_{\mathrm{MP}}$ - current at maximum power point $[\mathrm{A}]$, MPP $[\mathrm{W}]$ and $\eta[\%]$ were show in Figure 3.

Only for $\mathrm{I}_{\mathrm{SC}}$ and $\eta$ isolated lines we can observe ideal accordance of them with light intensity spatial distribution onto whole tested screen [10].

The highest values of light intensity, as we can notice, are placed in the field corresponding to the four upper rows (rows from $1^{\text {st }}$ to $4^{\text {th }}$ ). Such area is responsible for deterioration of the homogenous distribution of light intensity. 
a) $\mathrm{U}_{\mathrm{OC}}$

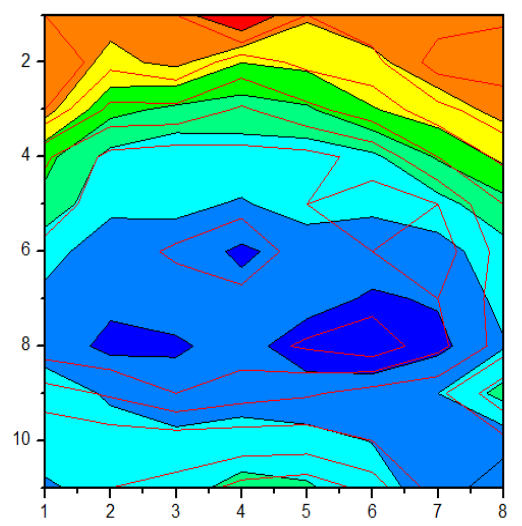

c) $U_{M P}$

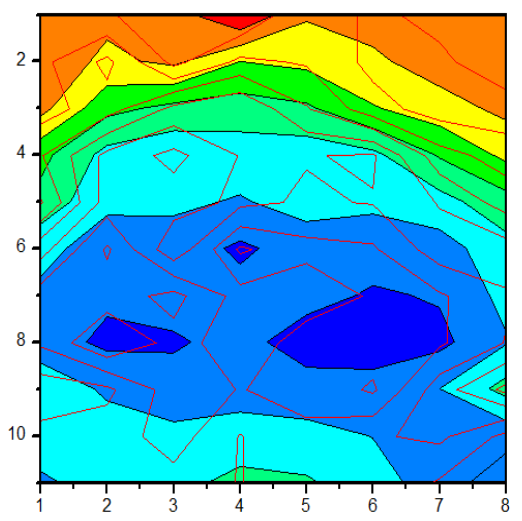

e) MPP

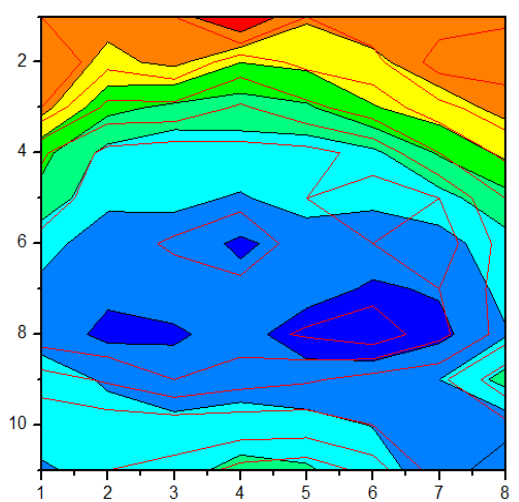

b) $\mathrm{I}_{\mathrm{SC}}$
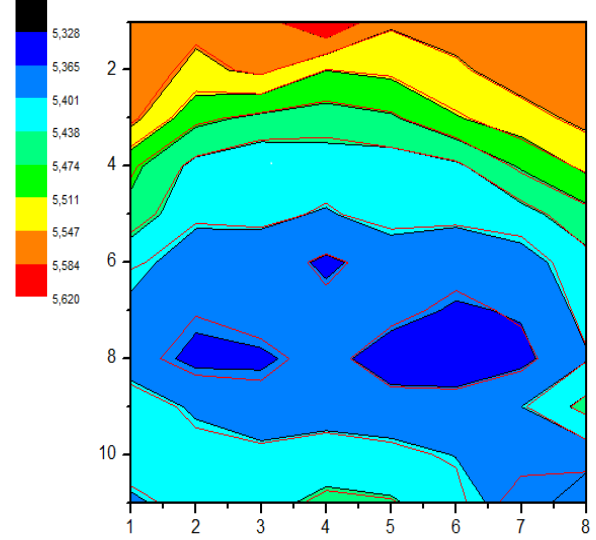

d) $I_{M P}$
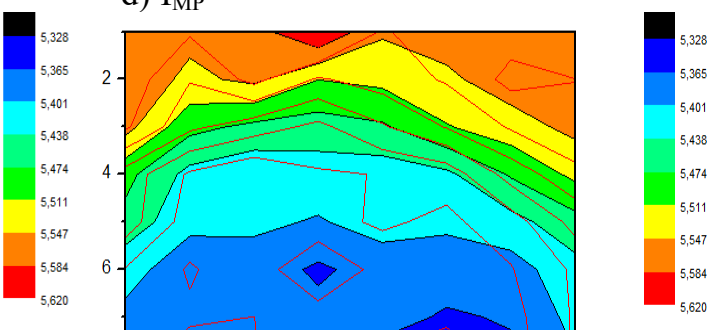

f) $\eta$
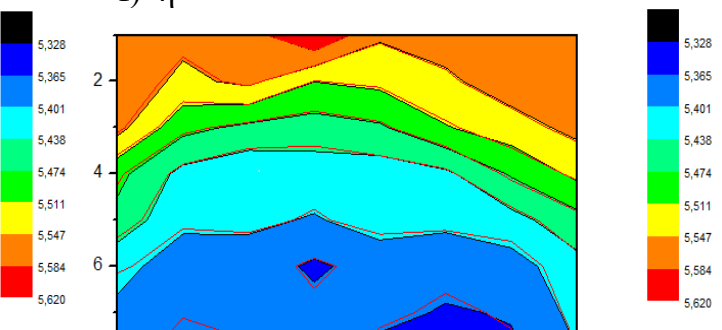

Fig. 3. Spatial distribution of light intensity on the area 8 column $x 11$ rows experimentally obtained from I-V characteristics of uniformity cell and putted on them isolated lines of a) $\mathrm{U}_{\mathrm{OC}}[\mathrm{V}]$, b) $\mathrm{I}_{\mathrm{SC}}[\mathrm{A}]$, c) $\mathrm{U}_{\mathrm{MP}}[\mathrm{V}]$, d) $\mathrm{I}_{\mathrm{MP}}[\mathrm{A}]$, e) MPP $[\mathrm{V}]$, f) $\eta[\%]$. 


\subsection{Non-uniformity parameter after testing screen reduction}

Further discussion for all investigated tested areas will be provided, for convenience, in mini tables similar to the Table 3. Please pay attention to the information given in the lower

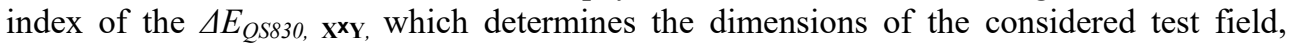
marked with different coloured rectangles in Table 4.

i) The non-uniformity condition for QS830A for the limited screen area, after exclusion of the mentioned four upper rows area, i.e. $0.825 \mathrm{~m} \times 1.58 \mathrm{~m}$ equal to $1.31 \mathrm{~m}^{2}$ from total screen area $3.59 \mathrm{~m}^{2}$ a matrix 8 columns $\times 7$ rows (area $2.28 \mathrm{~m}^{2}$ ), marked with a blue rectangle in Table 2 was designated.

\begin{tabular}{|c|c|c|c|c|}
\hline & $E_{\max Q S}[\mathbf{A}]$ & $\boldsymbol{E}_{\min Q S}[\mathbf{A}]$ & $\Delta E_{Q S 830 A}[1 / 1]$ & $\begin{array}{c}\left(\Delta E_{Q S 830} \pm\right. \\
\left.d\left(\Delta E_{Q S 8304}\right)\right)[\%]\end{array}$ \\
\hline$\Delta E_{O S 830 A}, 8^{x_{7}}$ & 5.465 & 5.331 & 0.01241 & $1.240 \pm 0.002$ \\
\hline
\end{tabular}

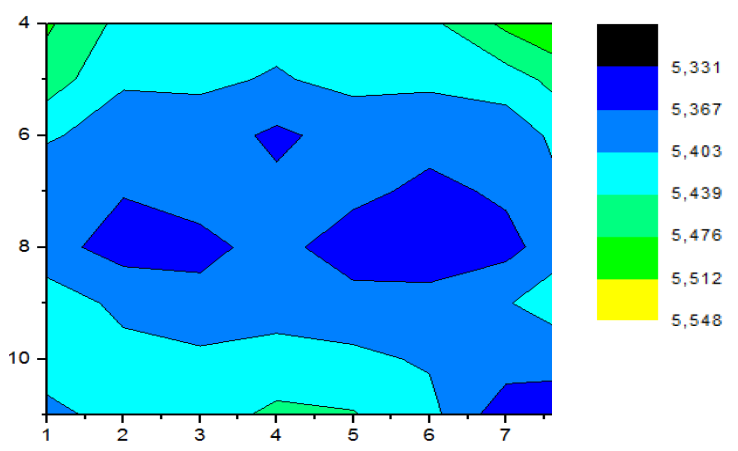

Fig. 4. Spatial distribution of light intensity on the area 8 column $\times 7$ rows experimentally obtained from I-V characteristics of uniformity cell [10].

The non-uniformity condition of QS830A for the new reduced tested area 8 columns $\times 7$ rows $\left(2.28 \mathrm{~m}^{2}\right)$ is real and is equal to $1.24 \%$.

$\triangle E_{Q S 830 A, \mathbf{X}^{\times} \mathbf{Y}}$ of QS830A for a three smaller than matrix 8 columns $\times 11$ rows areas: ii) $6 \times 9\left(2.20 \mathrm{~m}^{2}\right)$, iii) $4 \times 7\left(1.14 \mathrm{~m}^{2}\right)$, and iiii) $4 \times 4\left(0.65 \mathrm{~m}^{2}\right)$ is presented below.

Table. 4. I ISC $_{\text {C }}$ A average distribution of uniformity c-Si cell with marked three fields of 6 columns $\times 10$ rows, 4 columns $\times 7$ rows and 4 columns $\times 4$ rows.

\begin{tabular}{|l|l|l|l|l|l|l|l|}
\hline 5.573 & 5.560 & 5.570 & 5.620 & 5.554 & 5.564 & 5.572 & 5.574 \\
\hline 5.574 & 5.534 & 5.557 & 5.511 & 5.518 & 5.542 & 5.568 & 5.582 \\
\hline 5.556 & 5.483 & 5.465 & 5.456 & 5.469 & 5.506 & 5.533 & 5.560 \\
\hline 5.481 & 5.428 & 5.408 & 5.414 & 5.420 & 5.431 & 5.484 & 5.519 \\
\hline 5.458 & 5.408 & 5.410 & 5.400 & 5.413 & 5.410 & 5.422 & 5.465 \\
\hline 5.409 & 5.383 & 5.385 & 5.360 & 5.381 & 5.380 & 5.381 & 5.427 \\
\hline 5.369 & 5.369 & 5.380 & 5.375 & 5.377 & 5.358 & 5.373 & 5.412 \\
\hline 5.379 & 5.353 & 5.358 & 5.379 & 5.347 & & 5.356 & 5.400 \\
\hline 5.424 & 5.394 & 5.378 & 5.380 & 5.381 & 5.388 & 5.399 & 5.452 \\
\hline 5.418 & 5.415 & 5.411 & 5.423 & 5.411 & 5.399 & 5.387 & 5.381 \\
\hline 5.395 & 5.414 & 5.428 & 5.445 & 5.442 & 5.415 & 5.342 & 5.342 \\
\hline
\end{tabular}


ii)After reduction of the investigated testing area to the violet rectangle visible in Table 4, i.e. after removing the two first top rows, first left and last right columns, we can calculate the non-uniformity condition corresponding to that reduced area. In this way we can get the largest but reduced testing area dimension 6 columns on 9 rows, i.e. area $2.20 \mathrm{~m}^{2}$.

\begin{tabular}{|c|c|c|c|c|}
\hline & $\boldsymbol{E}_{\max Q S[\mathbf{A}]}$ & $\boldsymbol{E}_{\min Q S}[\mathbf{A}]$ & $\Delta E_{Q S 830 A}[1 / 1]$ & $\begin{array}{c}\left(\Delta E_{Q S 830} \pm\right. \\
\left.d\left(\Delta E_{O S 830 A}\right)\right)[\%]\end{array}$ \\
\hline$\Delta E_{O S 830 A},{ }_{6} \mathbf{x}_{9}$ & 5.568 & 5.331 & 0.01859 & $1.860 \pm 0.002$ \\
\hline
\end{tabular}

iii) After re-limiting the violet PV module testing area up to smaller one, i.e. after rejection two left and two right external columns, next first top row and the last bottom row, we will get matrix 4 column $\times 7$ rows $\left(1.14 \mathrm{~m}^{2}\right)$ area marked with a green rectangle in Table 4 .

\begin{tabular}{|c|c|c|c|c|}
\cline { 2 - 5 } \multicolumn{1}{c|}{} & $E_{\max Q S}[\mathrm{~A}]$ & $E_{\min Q S}[\mathrm{~A}]$ & $\boldsymbol{\Delta E}_{Q S 830 A}[\mathbf{1} / \mathbf{1}]$ & $\begin{array}{c}\left(\Delta E_{Q S 830} \pm\right. \\
\left.\boldsymbol{d}\left(\Delta E_{Q S 830 A}\right)\right)[\%]\end{array}$ \\
\hline$\Delta E_{Q S 830 A}, 4^{\mathrm{x}} 7$ & $\mathbf{5 . 4 3 1}$ & 5.331 & 0.00929 & $\mathbf{0 . 9 3 0 \pm 0 . 0 0 2}$ \\
\hline
\end{tabular}

iv) After re-limiting green testing area, i.e. after exclusion of the two top rows and last bottom row we achieved the 4 columns $\times 4$ rows $\left(0.65 \mathrm{~m}^{2}\right)$ area marked with a red rectangle in Table 4, the smallest area investigated at this paper.

\begin{tabular}{|c|c|c|c|c|}
\hline & $E_{\max Q \mathbf{S}}[\mathbf{A}]$ & $\boldsymbol{E}_{\min Q S}[\mathbf{A}]$ & $\Delta E_{Q S 830 A}[1 / 1]$ & $\begin{array}{c}\Delta E_{Q S 8304} \\
d\left(\Delta E_{O S 838}\right)[\%]\end{array}$ \\
\hline$\Delta E_{O S 830 A},{ }_{4} \mathbf{x}_{4}$ & 5.385 & 5.331 & 0.00504 & $0.504 \pm 0.004$ \\
\hline
\end{tabular}

Both mini areas exhibit a very good non-uniformity of light intensity parameter. We can measure the small photovoltaic modules on both of these limited separate areas of the test screen, assuring that the non-uniformity condition for module simulator Class AAA is met.

\section{Conclusions}

In the considered case, when after replacing the simulator light source - xenon lamp, for the entire measurement screen the condition of non-uniformity is not met. Before analyzing the repetition of the inhomogeneity tests it is worthwhile to carefully and particularly analyze the matrix of spatial distribution of light intensity. If the largest contribution to exceeding the admissible value of inhomogeneity defining a simulator Class A contributes to the upper row of the test field, it is enough to eliminate the field of recognition from the measurement area and analyze the data again. After changing the flush pulse lamp the entire $1.58 \mathrm{~m} \times 2.27 \mathrm{~m}$ size field does not meet the condition of non-uniformity for a Class A QS830A simulator. However, the reduced area of $1.185 \mathrm{~m} \times 1.857 \mathrm{~m}$ and the smaller fields considered in this work are already fulfilled. All of the smaller considered areas are perfectly fulfilling the non-uniformity condition for the QS830A Class AAA simulator. The heterogeneity improvement of the test field compared with the next reduction by individual rows and columns in its area are shown in Table 5. 
Table 5. QS830A non-uniformity parameter for investigated reduced tested area.

\begin{tabular}{|c|c|c|c|}
\hline $\begin{array}{c}\text { Reduced tested } \\
\text { area, } \\
\text { columns x rows }\end{array}$ & $\begin{array}{c}\text { Reduced tested area } \\
{\left[\mathbf{m}^{2}\right]}\end{array}$ & $\begin{array}{c}\text { Reduced tested } \\
\text { area / total tested } \\
\text { screen [\%] }\end{array}$ & $\begin{array}{c}\text { Non-uniformity } \\
\text { parameter for Class } \\
\text { A simulator [\%] }\end{array}$ \\
\hline $\mathbf{8} \times \mathbf{~ 1 1}$ & $1.58 \times 2.27=3.59$ & 79.8 & $\begin{array}{c}2.64, \text { the condition } \\
\text { is not met }\end{array}$ \\
\hline $\mathbf{6 \times 9}$ & $1.185 \times 1.857=2.20$ & 54.6 & 1.86 \\
\hline $8 \times 7$ & $1.58 \times 1.445=2,28$ & 61.3 & 1.24 \\
\hline $4 \times 1.445=1.14$ & 25.3 & 0.93 \\
\hline $4 \times 4$ & $0.79 \times 1.445$ & 0.50 \\
\hline
\end{tabular}

Placing a tested standard dimensions photovoltaic module (i.e. $1.1 \mathrm{~m} \times 1.6 \mathrm{~m}$ ) just below the second row from the top of the test screen we are sure that the non-uniformity condition for Class A QS830A is met. Testing a small photovoltaic module with an area of about $0.6 \mathrm{~m}^{2}$ a perfect uniformity of light intensity is guaranteed. It should be emphasized that we can act in such way only when testing smaller solar modules with an area of less than $2.2 \mathrm{~m}^{2}$ up to around $0.6 \mathrm{~m}^{2}$. Nevertheless, the best solution is to repeat the non-uniformity test with correct lamp setting until we achieve a guaranteed non-uniformity value of less than $2 \%$ for total tested screen. For each of the 88 segments, we repeat this test three times, it means we are performing the current-voltage characteristics of the uniformity c-Si cell a 284 times while determining the non-uniformity parameter of the light intensity for solar simulator.

Authors of the paper would like to thanks for support in this research to European Regional Development Fund and the Polish state budget within the Framework of the Carpathian Regional Operational Programme (RPPK.01.03.00-18-001/10-00) through the funding of the Center for Innovation and Transfer of Natural Science and Engineering Knowledge of the University of Rzeszow.

\section{References}

1. Website Endeas Oy Company http://www.endeas.fi.download/quicksun-800-series/

2. International Standard IEC 60904-9 Edition 2.0: Solar simulator performance requirements

3. International Standard IEC 60904-1 Edition 2.0: Cell and module current-voltage measurement procedure

4. International Standard IEC 60904-3 Edition 2.0: Measurements of current-voltage characteristics in the Standard Test Conditions STC

5. International Standard IEC 891 Edition 2.0: Irradiance and temperature correction procedures and coefficients

6. International Standard IEC 61853-1: Measurements of current-voltage characteristics in the Nominal Operating Cell Temperature Condition NOCT

7. C. Doroz, V. Fakhfouri, Y. Pelet, J. Roux, N. Peguiron, P.-R. Beljean, Proceedings of the 25th European Photovoltaic Solar Energy Conference and Exhibition, 5th World Conference on Photovoltaic Energy Conversion, 06-10 September 2010, Valencia, Spain, 3884-3888 (2010)

8. ASTM International West Conshohocken Patent ASTM E1084-86 (2015)

9. J. R. G. Ross, M. I. Smokler, Electricity from photovoltaic solar cells: Flat-Plate Solar Array Project final report (Springfield, NASA 2012)

10. R. Maciejko, The impact of uniformity of lighting on the quality of PV module's certificate (Faculty of Mathematics and Natural Sciences, University of Rzeszow, MSc thesis, Rzeszow 2017) 